\title{
Die kommunikative Verfertigung von Altersidentität
}

\author{
Reinhard Fiehler
}

Dieser Beitrag thematisiert Besonderheiten der sprachlichen Kommunikation im Alter. Zunächst werden allgemeine Charakteristika der Altersidentität skizziert, in der Komponenten wie Sozialität und Partner-bzw. Personenorienticrung eine besondere Rolle spielen. Anschließend werden Merkmale des Diskurses erörtert, in dem Altersidentität ausgebildet und fortentwickelt wird Vor allem die Ambivalenz zwischen dem Festhalten an der Erwachsenenidentität und dem Akzeptieren von Abweichungen davon ist für diesen Diskurs konstitutiv. Anhand exemplanischer Gesprächsausschnitte wird sodann aufgezeigt, in welcher Weise sich die für das Alter typischen Identitätskomponenten sowie die Orientierung an den Normalitätsstandards der mittleren Generation im kommunikativen Geschehen manifestieren.

\section{Abhängige Generationen}

Betrachtet man die individuelle Lebensspanne, so ist es eine geläufige Gliederung, sie in Jugend, Erwachsenenzeit und Alter zu unterteilen, und auch im gesellschaftlichen Maßstab ist es üblich, zwischen Jugend, Erwachsenen und Alten als koexistierenden Generationen zu unterscheiden. Ungeachtet feinerer Differenzierungen ist diese Dreiteilung alltagsweltlich fest im Bewusstsein verankert und besitzt eine starke orientierende Kraft. Trotz der kanonischen Reihenbildung, die von jedem Individuum - individuell wie gesellschaftlich - zu durchlaufen ist, sind diese drei Lebensalter bzw. Generationen hinsichtlich ihrer sozialen Bedeutung aber nicht gleichrangig. Beschränkt man die Betrachtung auf die drei koexistierenden Generationen, so besteht kein Zweifel, dass die mittlere, die Erwachsenengeneration, die zentrale und dominante ist. Sie ist Träger des gesellschaftlichen Reproduktionsprozesses, sowohl im ökonomischen wie auch im biologischen Sinne. Die beiden anderen Generationen sind in vielfaltiger Hinsicht von ihr abhängig und werden von ihr dominiert: Sie sind ihr gegenüber in einer marginalen Position. Das Verhältmis der Randgenerationen zur mittleren lässt sich am prägnantesten wohl durch ein ,noch nicht' bzw. ,nicht mehr' charakterisieren. 
Diese Situation kann nicht ohne Auswirkungen auf die Identitätsbildung der Individuen in den Randgenerationen bleiben: Sie erfolgt zu einem erheblichen Stück in der Auseinandersetzung mit den Normen, Werten und Leitbildern der Erwachsenengeneration, die zugleich die gesellschaftlich zentralen und präferierten sind. Unterschiedlich sind allerdings Strategien und Stoßrichtung der Auseinandersetzung: Die Jugendlichen verhalten sich in der äußeren Erscheinungsform vielfach oppositionell-distanzierend zur Erwachsenenidentität. Der Wunsch, anders zu sein oder zu werden, zielt auf eine alternative Identität und drückt Abgrenzung zur Erwachsenenwelt aus (vgl. Schwitalla, 1994). Die Alten hingegen verhalten sich eher partizipatorisch-affirmativ. In dem Wunsch, immer noch so zu sein wie die Erwachsenen, scheint ihr positiver Bezug zur Erwachsenenidentität auf. Eine Abgrenzung erfolgt hier gegen die alten Alten, die nicht mehr in der Lage sind, das Ideal der voll entwickelten erwachsenen Persönlichkeit für sich zu verwirklichen.

Versucht man zunächst die Erwachsenenidentität prototypisch zu charakterisieren, so sind als wesentliche Aspekte u.a. folgende Merkmale zu benennen, die zugleich normativ wirken:

- Eigenständigkeit und Individualität,

- Selbständigkeit und Selbstbestimmung,

- Kompetenz und Leistungsfahigkeit sowie

- Aufgaben- und Sachorientierung.

Sowohl die Jungen wie auch die Alten weichen von diesem Leitbild der entwickelten erwachsenen Persönlichkeit ab. Beide Gruppen sind der Tendenz nach weniger individualistisch orientiert, sondem präferieren einen höheren Grad an Gemeinschaftlichkeit bzw. Sozialität. Einher geht damit ein höherer Grad an Partner- und Personenorientierung, der mit der Aufgaben- und Sachorientierung der Erwachsenengeneration kontrastiert. Dabei kann man die Präferenz für Gemeinschaftlichkeit als Folge der marginalen Position verstehen: Sie bedingt ein Zusammenrücken und eine wechselseitige Unterstützung in der gemeinsamen inferioren Position, eine größere generationsinterne Solidarisierung (z.B. in Form von, peer groups'), die nach außen hin zugleich als Abgrenzung gegenüber den anderen Gruppen erscheint. Die marginale Lage erfordert identitätsstrukturell darüber hinaus die Produktion eigener Bedeutsamkeit, sowohl individuell wie auch als Gruppe.

Betrachtet man den Übergang von der Erwachsenen- zur Altersidentität, so bringt er demnach auf der einen Seite eine Abschwächung der Identitätskomponente ,Eigen- 
ständigkeit und Individualität' zugunsten von ,Gemeinschaftlichkeit und Sozialität' mit sich und auf der anderen Seite die Stärkung der Komponente ,Partner- und Personenorientierung ' anstelle von ,Aufgaben- und Sachorientierung'. Empirische Analysen in Abschnitt 4 werden versuchen, dies zu belegen.

Diese Überlegungen, die stark verallgemeinernd verfahren, um Grundstrukturen sichtbar werden zu lassen, machen deutlich, dass die Generationen nicht isoliert betrachtet werden dürfen, weil sie sich in nicht unerheblichem Ausmaß durch die wechselseitige Bezugnahme aufeinander konstituieren. So müssen auch die Alten in der Abfolge der Generationen als zwar erkennbar separierte, zugleich aber auch unauflöslich an die anderen Generationen gebundene und auf sie bezogene Gruppe gesehen werden, wobei eine isolierte Betrachtung wesentliche Konstitutionsmomente verfehlen würde. Für die Identitätsbildung der beiden Randgenerationen bedeutet dies, dass sie ganz wesentlich mit Bezug auf und in Abgrenzung zur mittleren Generation erfolgt.

\section{Alterskommunikation}

Grundlegend für die folgenden Ausführungen ist die Auffassung, dass die Erscheinungsform von Alterskommunikation nicht in erster Linie biologisch bestimmt ist, sondern vielmehr aus der Verarbeitung lebensgeschichtlicher Veränderungen und Erfahrungen und der Bearbeitung spezifischer sozialer Anforderungen und Aufgaben resultiert (vgl. Fiehler, 2002). Solche Veränderungen und Erfahrungen sind beispielsweise das Ende der Berufstätigkeit, der Dominanzverlust im Zuge der Generationsablösung, die zunehmenden körperlichen und mentalen Beeinträchtigungen oder die altersstereotype Behandlung durch Jüngere.

Diese und andere Veränderungen der Lebenssituation, die typischerweise mit wachsendem Alter eintreten, und die Erfahrungen, die beim Durchleben dieser Veränderungen gemacht werden, wirken sich in spezifischer Weise auf das sprachlichkommunikative Verhalten der betreffenden Personen aus. Das folgende Beispiel kann diese These verdeutlichen: Wird - z.B. als Folge zunehmender Immobilität oder eines wachsenden Desinteresses - die aktuelle Welterfahrung geringer, so bedeutet dies kommunikativ, dass vermehrt auf vergangene Erfahrungen zurückgegriffen werden muss, weil neue nicht zur Verfügung stehen. Der Anteil autobiographischer Erzählungen wird daher zunehmen. Sind aktuelle Fragen und Themen Gegenstand des Gesprächs, so kann darauf - je nach Verarbeitungsstrategie - unterschiedlich reagiert werden: Bei Interesse z.B. mit intensivem Nachfragen, um diese Erfahrungen, nach- 
zuholen', wobei das Gespräch Züge der Wissensvermittlung oder des Belehrens annehmen kann. Besteht hingegen kein Interesse, so kann dies bedeuten, dass der alte Mensch sich aus dem Gespräch ausblendet, oder aber, dass er versucht, das Thema in seinem Sinne zu beeinflussen (wie das z.B. ,zu seiner Zeit' war). Die zentrale Kompensationsstrategie für den Verlust aktueller Welterfahrung besteht in der Medienrezeption, so dass Berichte und Erzählungen über die gesehenen Sendungen zu einem wichtigen Bestandteil des Kommunikationsaufkommens werden.

Alterstypische Veränderungen und Erfahrungen strukturieren auf diese Weise den kommunikativen Haushalt (Luckmann, 1988) in quantitativer wie in qualitativer Hinsicht um. Quantitative Veränderungen können in der Zunahme (Verbosität, vgl. Ryan \& Kwong See, 1998: 59-61), aber auch in der Abnahme des Kommunikationsaufkommens bestehen. Die qualitativen Veränderungen liegen zum einen auf der thematischen Ebene in dem Sinn, dass die typischen sozialen Veränderungen und Erfahrungen häufig Gegenstand von Gesprächen sind. Sie betreffen aber auch Vorkommen und Quantität bestimmter Gesprächsformen (z.B. Erzählen, Klatsch), bestimmter kommunikativer Muster (z.B. empathische Realisienungen des Musters der Bewertungsteilung) und kommunikativer Strategien (z.B. Stilisierung als .alt', Einbringen einer Vergangenheitsperspektive). Sie berühren ferner äußerungsstrukturelle und gesprächsorganisatorische Aspekte wie den Partnerzuschnitt von Äußerungen, die Bezugnahme auf Vorgängeräußerungen oder die Gestaltung thematischer Kohärenz (z.B. assoziative Anschlüsse). Die Umstrukturierung des kommunikativen Haushalts bleibt dabei auch nicht ohne Auswirkungen auf die Ebene der sprachlichen Mittel. Veränderungen und Erfahrungen konturieren so ein Kommunikationsverhalten, das man als Stil des Alters bezeichnen kann. Er ist Resultat der kommunikativen Auseinandersetzung mit den Lebensbedingungen im Alter (vgl. Fiehler, 1997; 2001).

Dabei ist klar, dass alterstypische Sprache und Kommunikation nicht Folge der Veränderung eines Faktors ist. Alle erlebten Veränderungen und Erfahrungen wirken, wenn sie eintreten, zusammen, und ihre jeweiligen kommunikativen Folgen interferieren. Auf der Ursachenseite sind also in der Regel Bündel von Faktoren anzusetzen, wobei diese Faktoren bei der einzelnen Person bzw. bei Personengruppen in je individuellen Konstellationen auftreten und zusammenwirken und zudem jeweils unterschiedliches Gewicht besitzen können. Die beschriebenen kommunikativen Auswirkungen sind natürlich nicht auf das Alter beschränkt. In dem Maße, wie entsprechende Veränderungen und Erfahrungen auch bei jüngeren Menschen vorliegen, finden sich 
auch dort z.B. autobiographische Erzählungen, Klatsch und das Hinzufügen einer Vergangenheitsperspektive, aber in anderer Frequenz und z.T. auch anderer Qualität. Das Alter zeichnet sich dadurch aus, dass diese kommunikativen Folgen aufgnund der Massierung der Veränderungen und Erfahrungen kumulieren und zu dem Eindruck einer spezifischen, konturierbaren Kommunikationsform führen.

\section{Altersidentităt}

\subsection{Die Ausbildung von Altersidentität}

Indem ältere Menschen diese Veränderungen, die gegenüber ihrer Situation als Erwachsene eintreten und die sie von dieser Phase entfernen, kommunikativ be- und verarbeiten, sind sie - nicht unbedingt intentional - zugleich noch mit einer weiter reichenden und umfassenderen Aufgabe befasst, der Ausbildung von Altersidentität. Weite Strecken der Gespräche, die alte Menschen untereinander oder mit jüngeren führen, lassen sich als - häufig impliziter - Identitätsdiskurs interpretieren, in dem es um die Ausarbeitung von Aspekten der Altersidentität geht. Dies geschieht in verschiedenen Gesprächsformen, wobei Erzählungen einen zentralen Platz einnehmen (Schiffrin, 1996), und mittels eines spezifischen Repertoires von kommunikativen Verfahren der Identitätskonstitution.

Die Ausbildung von Identität ist ein kontinuierlicher Prozess (Keupp et al., 1999: 190): „Der Identitătsprozeß ist, so sehen es die meisten neueren Ansätze der Identitätsforschung, nicht nur ein Mittel, um am Ende der Adoleszenz ein bestimmtes Plateau einer gesicherten Identität zu erreichen, sondern der Motor lebenslanger Entwicklung."

Die Ausbildung von Identität ist darüber hinaus ganz wesentlich ein interaktiver Prozess (Androutsopoulos, 2001: 62): „Identität ist nicht etwas Vorgegebenes, Fixes und Unveränderliches, sondern eine ,emergent construction', das Ergebnis eines rhetorischen Produktions- und Interpretationsprozesses [...], und wird im Gespräch durch die Beteiligten gemeinsam konstituiert.“

Die gemeinschaftliche Konstitution und Prozessierung von Identităt ist Bestandteil jedweder Interaktion, sie kann dabei allerdings unterschiedlich stark im Vordergnund stehen bzw. thematisch sein.

Die wechselseitige Ausbildung von Identităt in der Interaktion umfasst für jeden der Beteiligten drei systematisch aufeinander bezogene Teilaufgaben: erstens Selbstpräsentation, zweitens Stellungnahme zur Selbstpräsentation der anderen Person, und 
drittens Reaktion auf die Stellungnahme der anderen Person zur eigenen Selbstpräsentation. Jeder Beitrag zur Interaktion enthält Aspekte der Selbstpräsentation. Zugleich nimmt man mit jedem Beitrag - wie indirekt auch immer - bewertend Stellung zur Selbstpräsentation der anderen Interaktionspartner. In Reaktion auf die Art der Stellungnahme der anderen Person zur eigenen Selbstpräsentation erfolgt dann eine Bekräftigung oder eine Modifikation dieser Präsentation. Da alle Beteiligten diese drei Teilaufgaben bearbeiten und da sie vielfaltig miteinander verflochten sind, wird deutlich, dass die Ausbildung von Identität in wechselseitiger Abhängigkeit voneinander erfolgt.

Die Konstitution und Prozessierung von Identität ist dabei sowohl ein interaktiver wie auch ein kommunikativer Prozess, der sich aller Kommunikationskanäle bedienen kann (verbal, prosodisch, nonverbal). Alle drei Teilaufgaben implizieren kommunikative Akte, wobei jede Teilaufgabe in der Interaktion durch verbale Äußerungen thematisch explizit bearbeitet werden kann. Identitätsbildung erfolgt so in und durch Kommunikation und kommt zugleich in ihr zum Ausdruck (Holstein \& Gubrium, 2000; Kraus, 1996).

\subsection{Manifestationen im Kommunikationsverhalten}

Kommt man zurück auf die eingangs getroffene Feststellung, dass Altersidentität zu wesentlichen Teilen in der Auseinandersetzung mit der Erwachsenenidentität ausgebildet wird, so ist zu erwarten, dass in der Kommunikation älterer Menschen drei Komplexe eine wichtige Rolle spielen:

- erstens vielfältige Formen des Nachweises, dass man dem Leitbild der erwachsenen Persönlichkeit (noch) entspricht,

- zweitens der Umgang mit eigenen Abweichungen von diesem Bild, und

- drittens die Konturierung eigenständiger Merkmale von Altersidentität.

Zum ersten Punkt: In dem Maße, wie die Erwachsenenidentität keine Selbstverständlichkeit mehr ist, wird es bedeutsam, im Gespräch mit Gleichaltrigen oder Jüngeren nachzuweisen, dass und welche Aspekte von Erwachsenenidentität unverändert vorliegen. Der Altersdiskurs ist so zu einem nicht unwesentlichen Teil ein Nachweisführen hinsichtlich der persönlichen Eigenständigkeit, Vollwertigkeit, Bedeutsamkeit, Kompetenz, Mobilität, Normalität etc. Die Darstellung dieser Eigenschaften geschieht aber nicht unmarkiert und selbstverständlich, sondern sie bekommt einen demonstrativen Charakter. Die Darstellung erfolgt z.B. mit einer gewissen Ausführlichkeit und Nachdrücklichkeit. 
Ein wichtiges kommunikatives Verfahren in diesem Nachweisdiskurs ist die Kontrastierung eigener Möglichkeiten mit denen anderer Alter, die über entsprechende Eigenschaften nicht mehr verfügen. Während der Nachweis, über relevante Merkmale der Erwachsenenidentität zu verfügen, in der Generation der Alten vorwiegend diskursiv geführt wird. geschieht dies in der Jugendgeneration auf vielfaltige Weise auch symbolisch, indem Attribute der Erwachsenenwelt übernommen werden (Rauchen, Alkohol trinken, Kleidung).

Zum zweiten Punkt: Die Feststellung, dass man in verschiedener Hinsicht dem Bild des Erwachsenen nicht mehr entspricht, erfolgt kommunikativ in Form der Thematisierung und des Beklagens dieser Abweichungen. Hierhin gehören alle Formen des ,painful self disclosure', wie sie von Coupland, Coupland und Giles (1991) beschrieben worden sind. Insbesondere geschieht dies in Gestalt des Krankheitsdiskurses oder des Konstatierens von nachlassenden Fähigkeiten.

Zum dritten Punkt: Einen wichtigen Raum nimmt aber auch die Darstellung von spezifisch neuen Identitätsaspekten ein, mit denen sich die ältere Generation von der mittleren absetzt. Hierzu gehört vor allem die stärkere Parıner- und Personenorientierung, die u.a. möglich wird, weil zentrale Aufgaben (Beruf, Kindererziehung) entfallen. Der Tendenz nach wird die dort vorherrschende Aufgaben- und Sachorientierung durch eine stärkere Personenorientierung ersetzt, die darin besteht, sich mehr für andere Personen zu interessieren, an ihnen Anteil zu nehmen und intensiver auf sie einzugehen (auch wenn dies häufig nur stereotyp geschieht). Sie findet in vielfältigen Formen kommunikativer Kooperativitat und wechselseitiger (Interstutzung Ausdruck, aber auch im wechselseitigen übereinander Reden (Klatsch). Das hohe Ausmaß an wechselseitiger Unterstützung kontrastiert deutlich mit der in der Erwachsenengeneration vorherrschenden Präferenz zur Selbstvertretung (vgl. Schmitt, 1997). Während ein Unterstützen bei Erwachsenen konkrete Anlässe in der Interaktion erfordert und an sie gebunden ist, kann es bei älteren Menschen einen fast habituellen Charakter haben. Ein weiteres Moment ist das kommunikative Zur-Geltung-Bringen des erworbenen Wissens (Erfahrungsschatz, Altersweisheit) und von Altersgelassenheit. Auch die Fähigkeit, Beeinträchtigungen und Defiziten positive Seiten abgewinnen zu können, kann zu diesen neuen Identitätsmerkmalen gehören.

Die zentrale Schwierigkeit der Entwicklung von Altersidentität besteht darin, nicht einfach nur weiter am Erwachsenenbild festzuhalten, sondern abweichende Identitätsaspekte auszubilden und sie zu integrieren, d.h. ein Selbstbild und Selbstverständnis zu 
entwickeln, das nicht nur eine Verlängerung der Erwachsenenidentität ist. Identitätsentwicklung im Alter bedeutet also, einerseits an zentralen Aspekten der Erwachsenenidentität (so lange wie möglich) festzuhalten, andererseits aber auch einige Aspekte aufzugeben und darüber hinaus neue Identitätsmerkmale zu entwickeln und zu integrieren. Prägend ist damit eine Ambivalenz zwischen dem Festhalten an den Standards und Werten der mittleren Generation und der Akzeptanz von Verändenungen und ihrer produktiven identitätsstrukturellen Verarbeitung. Identitätsarbeit im Alter hat, insofern die drei genannten Aspekte zu vermitteln sind, den Charakter einer konfliktbezogenen Aushandlung (Keupp et al., 1999: 216): „Dabei werden unterschiedliche Selbsterfahrungen nicht im Sinne eines auf Widerspruchsfreiheit angelegten Gleichgewichtsmodells aufgelöst, sondem in ein dynamisches Verhältnis gebracht, das treffender als konfliktorientierter Spannungszustand beschrieben werden kann.“

\section{Beispielanalyse}

Die Prozesse der kommunikativen Konstitution und Prozessierung von Altersidentität sollen im Folgenden beispielhaft an Ausschnitten aus einem authentischen Gespräch verdeutlicht werden. Es handelt sich um ein Tischgespräch, das bei einem gemeinsamen Essen in einem griechischen Restaurant geführt wird. Das Ehepaar Kellner (KM und $K F$ ) hat Herm Frahm (F) eingeladen, um mit ihm den 75. Geburtstag von Herrn Kellner nachzufeiern, an dem er nicht hatte teilnehmen können. Die drei - alle über 70 - haben bis vor kurzem gemeinsam in einem Orchester musiziert. An dem Essen nimmt auch die Sprachwissenschaftlerin Frau Seebohm (S) teil, die bereits mehrere längere Gespräche dieser drei befreundeten Personen aufgenommen hat und die auch dieses Tischgespräch aufzeichnet. Gegenstand des ersten Teils des Gesprächs sind detaillierte Schilderungen von Herrn und Frau Kellner über den Ablauf der Geburtstagsfeierlichkeiten, die sich über mehrere Tage erstreckten.

\subsection{Nachweis von Erwachsenenidentität}

Herr und Frau Kellner bemühen sich, in dem Gespräch zu zeigen, dass sie die Geburtstagsfeier kompetent vorbereitet und durchgeführt haben. Dazu gehört auch, dass sie in dem Hotel, in dem die Feier stattfand, übernachtet haben: 


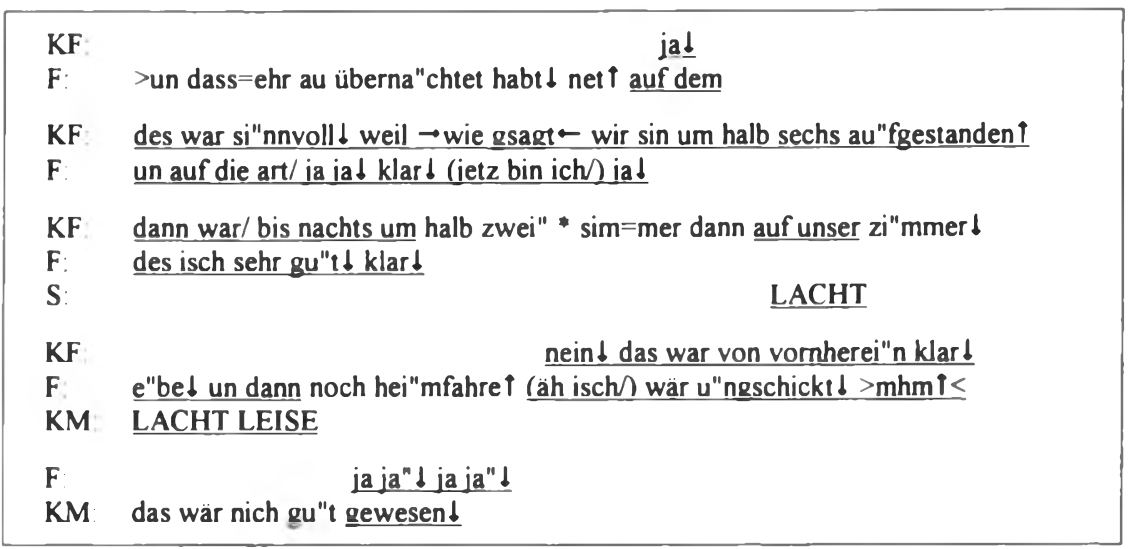

Frau Kellner betont in dieser Sequenz zum einen das Durchhaltevermögen des Ehepaars am Geburtstag. Zum anderen wird dadurch, dass sie die Übernachtung als sinnvoll und von vornherein klar charakterisiert, die eigene Planungs- und Handlungskompetenz demonstriert.

Die Selbstdarstellung als kompetent handelnde Erwachsene wird dadurch unterstützt, dass unmittelbar im Anschluss diejenigen Gäste thematisiert werden, die im Kontrast dazu nicht im Hotel übernachtet, sondern die Feier verlassen haben:

\begin{tabular}{|c|c|}
\hline KF: & wie gsagt messmers $\uparrow * \leftarrow$ äh $\rightarrow \mathrm{s} /$ \\
\hline KF & messmer senior wurde von messmer ju"nior heim gefahren $\uparrow$ \\
\hline S: & $>$ SCHMUNZELT $<$ \\
\hline $\mathrm{F}$ & ah so" $\downarrow$ die" sin heim gfahre $\downarrow$ noch in de na"cht dann $\uparrow$ \\
\hline KM & ia iat \\
\hline KF: & ia ia $\downarrow$ un da er/ wir ham=s a"ngeboten/ nei $n \downarrow$ \\
\hline $\mathbf{F}$ & also um zwei" $\downarrow$ nachts $\downarrow$ \\
\hline KM: & nee die sind e"her $\downarrow$ \\
\hline F & sind sie vo"rzeitie gegangel ah iat ah so" \\
\hline $\mathrm{KF}$ : & die sind/ die sind so um mi"tternacht * gfahrn \\
\hline $\begin{array}{l}\mathrm{F} \\
\mathrm{KM}\end{array}$ & aber au" spät $\downarrow$ iaha $\underset{\text { ia ja } \uparrow}{\text { (ia sicher } \downarrow \text { ) }}$ \\
\hline
\end{tabular}

Messmer senior hat nicht nur nicht bis zum Ende der Feier durchgehalten (obwohl Herr Frahm ihm immerhin zugesteht, dass es auch spät war, als er ging), sondern er musste auch die Hilfe der Erwachsenengeneration in Gestalt von Messmer junior in 
Anspruch nehmen. Die Messmer-Episode wird von den Kellners zu einer positiven Selbstdarstellung genutzt, indem sie kontrastiv verdeutlichen, dass Messmer senior nicht - wie sie selbst - selbstbestimmt agiert, sondem sich in Abhängigkeit von Messmer junior begibt. Er wird so von den Kellners als kategorial den alten Alten zugehörend behandelt.

Auch der nächste im Gespräch behandelte Gast hat nicht am Ort der Feier übernachtet: $K F$ : und gu"drun und der bernd ham dann die mutter noch * nach o"ffenburg gebracht I weil sie in i"hrem belt schlafen wollte d. Die fehlende Flexibilität der Mutter hebt wiederum die Flexibilität der Kellners hervor. Erst im Anschluss daran werden die Personen besprochen, die vor Ort übernachtet haben: $K F$ : und wie gesagt un marIi"na I un der ernst mar"schall I die ham übernachtel I un mein schwager und meine schwägerin t. Es ist deutlich, dass Herr und Frau Kellner sich durch diese Polarisierung demonstrativ auf der Seite der kompetenten Erwachsenen positionieren, die es verstehen, situationsangemessen und flexibel zu agieren.

Der betrachtete Abschnitt des Gesprächs kreist nicht explizit thematisch, aber indirekt in seiner Funktion für die Identitätsarbeit der Beteiligten darum, nachzuweisen, dass die Kellners, obwohl der besondere Geburtstag ein nicht unerhebliches Alter signalisiert, nach wie vor über wesentliche Aspekte der Erwachsenenidentität wie Kompetenz und Leistungsvermögen verfügen. Er verweist damit zugleich auf die Notwendigkeit und das Problem, analytisch von der Vielfalt der Oberflächenthematiken zu den grundlegenderen Thematiken identitätsstruktureller Art vorzustoßen.

In einer weiteren Episode des Gesprächs wird Teilhabe an den normalen Routinen des Erwachsenenlebens und Mobilität demonstriert. Frau Thomas (T), die Besitzerin des Restaurants, weist, als sie die Gruppe bedient, darauf hin, dass zu einem bestimmten Termin dort eine Musikgruppe spielen wird. Herr und Frau Kellner stellen bedauemd fest, dass sie an dieser Veranstaltung nicht teilnehmen können, weil sie zu diesem Zeitpunkt verreist sind.

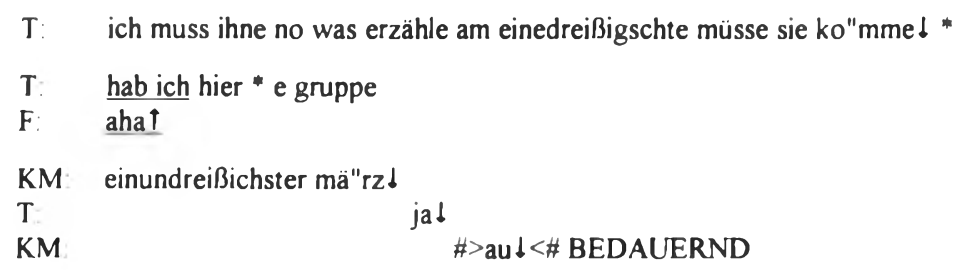




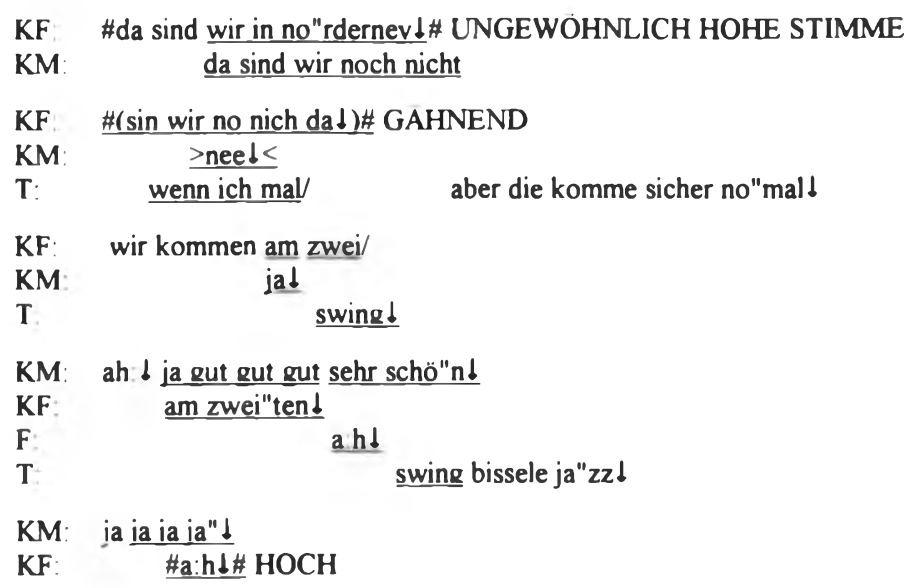

Herr und Frau Kellner stellen fest, dass sie zum fraglichen Zeitpunkt in no"rderney bzw. no nich da sind. Obwohl dies unter sachlichen Gesichtspunkten ausreichen würde und Frau Thomas sich damit zufrieden zu geben scheint (aber die komme sicher no"mal $)$, haben die Kellners und Herr Frahm eine solche sachlich-ökonomische Orientierung nicht und thematisieren mit übergroßer Detaillienung Termin und Modalitäten der Reise:

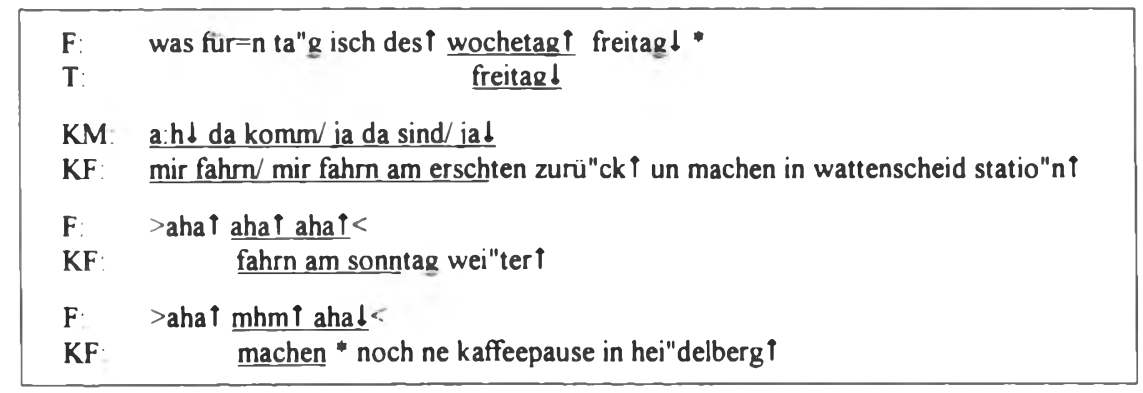

Allein mit Höflichkeit lässt sich die vorfindliche Breite und ,Umständlichkeit', die hier nur teilweise wiedergegeben ist, nicht erklären. Die Ausführlichkeit der Darstellung signalisiert, dass nicht nur das Terminproblem behandelt wird, sondern dass es genutzt wird, um noch etwas Weiteres zu demonstrieren. Die Funktionalität der Kommunikation liegt nicht allein im Sachlichen, sondern ist identitätsstrukturell begründet: Die Beteiligten verdeutlichen, dass sie ausgefullt sind und nicht jeden angebotenen Termin wahmehmen können, dass sie nicht ortsgebunden, sondern mobil sind und dass sie 
eine ganz ,normale' Reise unternehmen, die sie selbst organisieren (es also keine speziell für Alte vororganisierte Fahrt ist) und gestalten (durch verschiedene Stationen auf der Rückreise). Kurz: Sie demonstrieren Kompetenz und Mobilität als Bestandteile der Erwachsenenidentität. Die Ausführlichkeit, die sich daraus ergibt, dass dies zusätzlich zur Bearbeitung des Sachproblems geschieht, kann aus der Perspektive von Erwachsenen irritierend sein und aufdringlich wirken und so das Stereotyp der Verbosität alter Menschen konstituieren oder stützen.

Auch Herr Frahm versucht, Aspekte der Erwachsenenidentität zu profilieren, indem er sich - aus seiner Perspektive - technisch Neuem gegenüber als aufgeschlossen darstellt: Nachdem Gudrun die Mutter heim in ihr eigenes Bett gebracht hat, hat sie ein Fax geschickt, dessen Inhalt Herr Kellner wiedergibt als: Wir sind alle gut angekommen. Herr Frahm nutzt dies, um aus der Darstellung der Ereignisse auszusteigen und das Faxgerät als eigenstăndiges Thema zu etablieren:

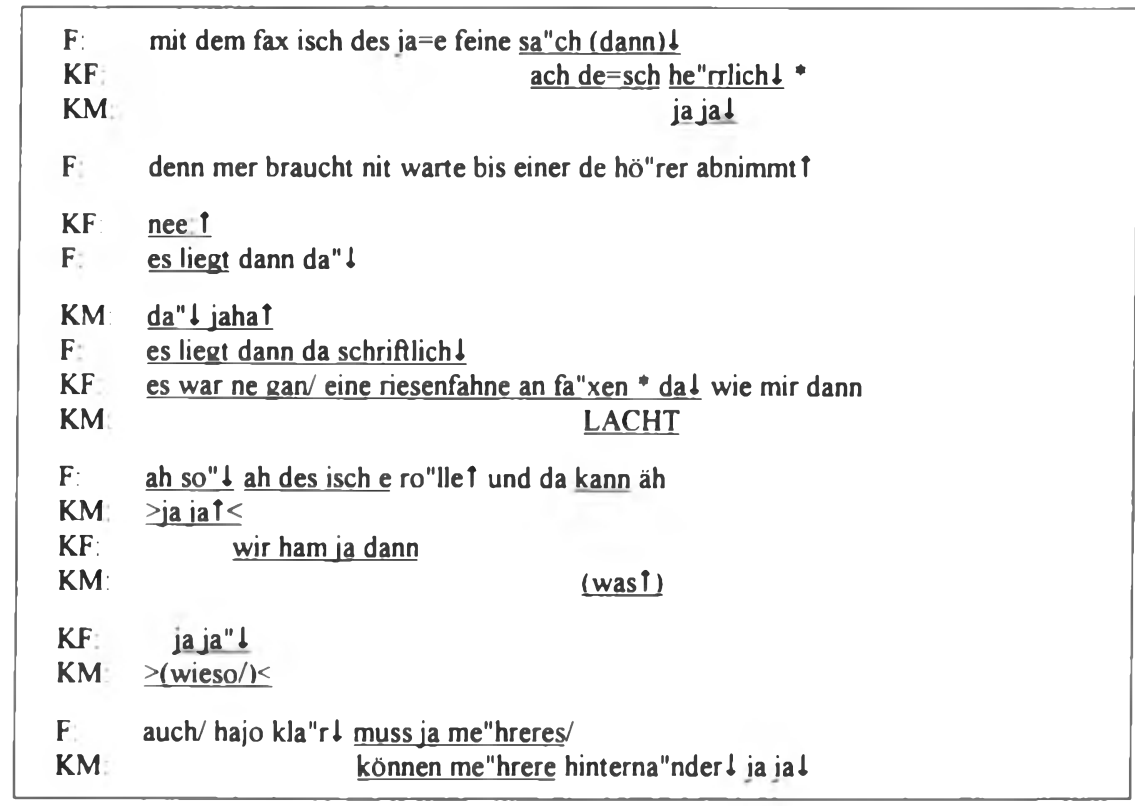

Die formelhafte Äußerung mit dem fax isch des ja=e feine sa"ch (dann) $\downarrow$, der sich Frau Kellner mit einer Bewertungsteilung anschließt und der auch Herr Kellner zustimmt, schafft potenziell Raum für eine längere kommunikative Behandlung dieses Geräts, mit der Herr Frahm sich möglicherweise als technisch aufgeschlossen darstel- 
len will. Die Tatsache aber, dass er zu dem Zeitpunkt, an dem das Gespräch stattfindet (Anfang 2000), ein Faxgerät und seine Leistungen fur so detailliert behandelnswert hält, lässt ihn als nicht auf der Höhe der Zeit - und das heißt eben tendenziell als alt erscheinen. Ein entsprechender Diskurs wird in der Erwachsenengeneration zu diesem Zeitpunkt schon über neuere Kommunikationstechnologien geführt. Auch dass Herr Frahm kein klares Bild von Faxpapierrollen hat (ah so" $\downarrow$ ah des isch e ro"lle $\uparrow$ ), trägt zum Scheitern seiner Darstellung von technischer Kompetenz bei. Hier misslingt die Darstellung von Erwachsenenidentität.

Die Versuche, Erwachsenenidentität nachzuweisen, unterliegen insgesamt einem strukturellen Dilemma. Der demonstrative Charakter des Nachweises macht deutlich, dass diese Identität für die Betroffenen nicht (mehr) selbstverständlich ist. Er verdeutlicht aber auch, dass die betreffenden Personen meinen, diesen Nachweis nötig zu haben: Der Versuch zu demonstrieren, nicht alt zu sein, macht gerade als alt kenntlich.

Die zweite der oben benannten Komponenten des Identitätsdiskurses älterer Menschen, der Umgang mit Abweichungen von der Erwachsenenidentität, ist in der Literatur verschiedentlich beschrieben worden, so dass hier gleich zu eigenständigen Aspekten der Altersidentität übergegangen werden soll.

\subsection{Partner- und Personenorientierung}

$\mathrm{Zu}$ den sich entwickelnden Identitätsaspekten, die das Alter von der Erwachsenenidentität unterscheiden, gehört in vielen Fällen eine Steigenung der Partner- und Personenorientierung, die sich $\mathrm{u} . \mathrm{a}$. in einem hohen $\mathrm{Ma}$ an kommunikativer Kooperativität und wechselseitiger Unterstützung im Gespräch äußert. Im Folgenden sollen zunächst Erscheinungsformen kommunikativer Kooperativität in einem engeren Sinne vorgestellt werden, um anschließend weitere Ausdrucksformen der verstärkten Partner- und Personenorientienung darzustellen.

Kommunikative Kooperativität (vgl. Fiehler, 1999: 54-56) zeigt sich im untersuchten Gespräch in einer Vielzahl von sprachlich-kommunikativen Phänomenen, die nachfolgend zusammengestellt und an einigen wenigen Beispielen illustriert werden sollen. Das AusmaB der kommunikativen Kooperativität führt dazu, dass das Sprechen an manchen Stellen den Charakter des gemeinsam miteinander Musizierens annimmt. Wie auch schon die bisher vorgestellten Transkriptausschnitte zeigen, ist simultanes oder überlappendes Sprechen eine überaus häufige und hervorstechende Erscheinung in diesem Gespräch. Weitere Merkmale kommunikativer Kooperativität sind: 
- Exzessiver Gebrauch von Rezeptions-/Bestätigungssignalen

KF un musst ja au en zi"mmer bstellt werde $\uparrow$ un für marti"na noch en

$F$ : $\quad$ ja ja ja ja $\downarrow$

KF: zimmer bestellt un schwager un schwä"gerin

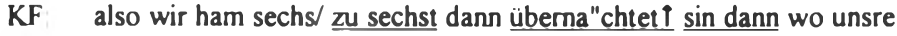

F $\underline{\mathrm{mhm} I} \underline{\mathrm{mhm}} \mathrm{mhm}$

Rezeptionssignale treten ungewöhnlich häufig multipel (als Batterie) und/oder gehäuft (voneinander abgesetzt, aber mehrfach auf engem Raum) auf. Sie gewinnen durch ihre Zahl zunehmend den Charakter von Bestätigungssignalen.

- Wechselseitige Paraphrasen (simultan bzw. sukzessive gesprochen)
F: des kann aber nit * dieses freibure hat nix zu tu"nl
KM: das hat damit nix zu tu"nd
KM: hat damit nix zu tun $\downarrow$ nein das hat damit ni"chts zu tun
F: $\quad$ ebel ebed ah iat ahial

In diesem Beispiel paraphrasiert zunächst Herr Kellner Herrn Frahm simultan, wobei KM die Äußerung von F vervollständigt. Danach wiederholt Herr Kellner seine Äußerung leicht variiert zweimal. Diese Wiederholungen begleitet dann $\mathrm{F}$ seinerseits durch eine Batterie von Bestätigungssignalen. Dieses Beispiel kann zugleich als Beleg für die identische oder variierende Wiederholung von Äußerungen durch den gleichen Sprecher dienen.

- Reformulierungen

$\mathrm{KM}$ : nee die sind $\mathrm{e}$ "hert

F: $\quad$ sind sie vo"rzeitio gegange

Reformulierungen unterscheiden sich von den Paraphrasen dadurch, dass sie das sprachliche Material der Bezugsäußerung stärker variieren. Dadurch sind sie in der Lage, neue Aspekte ins Spiel zu bringen. In diesem Fall wird das neutrale eher zu vorzeitig verschärft: Auf diese Weise wird das nicht Normgerechte des Zeitpunkts betont. Auch Reformulierungen können, wie in diesem Fall, simultan zur Bezugsäußerung oder im Anschluss an sie realisiert werden (zu Paraphrasen und Reformulierungen vgl. auch Sachweh, 2001). 
- Gemeinschafiliche Außerungsproduktion

\begin{tabular}{|ll|}
\hline $\mathrm{F}:$ & die mutter von de gu"drun \\
$\mathrm{KF}$ & von der gu"drun \\
$\mathrm{KF}:$ & isch die $\uparrow$ \\
$\mathrm{F}:$ & von von meinem verstorben ve"tter $\mathrm{mhm} \uparrow$ \\
\hline
\end{tabular}

Herr Frahm beginnt eine Äußerung mit die mutter von de gu"drun isch die $\uparrow$ und Frau Kellner setzt sie mit frau von meinem verstorbenen vetter fort, so dass es zu einer gemeinschaftlichen Äußerungsproduktion kommt (vgl. Schwitalla, 1992; Sachweh, 2001). In beiden Äußerungshälften gibt es zudem jeweils Paraphrasen von Äußerungsteilen durch den gerade nicht dominanten Sprecher, die zum Teil simultan formuliert werden - ein ausgesprochen dichtes Beispiel für kommunikative Kooperativität.

- Entwickelndes Nachfragen

$$
\begin{aligned}
& \text { KF : die äh mu"tter von der gu"drun isch die frau meines verstorbenen ve"tters } \\
& \text { KF. der ja nach=m tod von } d=n \text { eltern war der ja mein vo"rmund } \downarrow \\
& \text { F } \quad \text { un die le"bt aber nochl iahal }
\end{aligned}
$$

Eine hohe Rate an Nachfragen ist ein weiteres Element kommunikativer Kooperativität. Sie signalisiert und demonstriert das Interesse des Hörers am Thema. Die Nachfragen führen in der Regel zu Detaillierungen, durch die die jeweilige Thematik weiter entwickelt und vertieft wird.

- Präferenz für Zustimmung

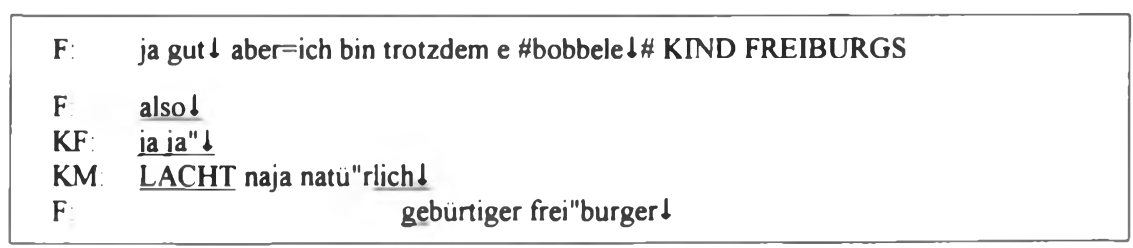

Die Gesprächspartner stellen fest, dass das Ehepaar Kellner insgesamt schon länger in Freiburg gelebt hat als Herr Frahm. F reklamiert daraufhin - quasi als rituellen Ausgleich -, dass er im Gegensatz zu den Kellners gebürtiger Freiburger ist. Das wird ihm sowohl von KF wie auch von KM zustimmend eingeräumt. Ähnlich wie in dieser Sequenz zeigt sich auch generell eine Tendenz, lieber zuzustimmen als zu problematisieren, zu relativieren oder zu widersprechen. 
- Bewertungsteilungen

F: $\quad$ mit dem fax isch des ja=e feine sa"ch (dann) $\downarrow$

$\mathrm{KF}$

ach de=sch he"rrlich

$\mathrm{KM}$

jaja!

Ein Spezialfall von Zustimmungen sind Bewertungsteilungen, bei denen die Beteiligten sich in demonstrativer Weise versichern, dass sie in der Bewertung eines Sachverhalts oder einer Person übereinstimmen (vgl. Fiehler, 1990: 221-225). In diesem Fall wird Herm Frahms positive Bewertung von Faxgeräten sowohl von Frau wie auch von Herrn Kellner gestützt. Indem Bewertungsteilungen manifest machen, dass die $\mathrm{Ge}-$ sprächspartner in relevanten Bewertungen übereinstimmen, haben sie eine stark gemeinschaftsstiftende und -stabilisierende Funktion.

- Stichwortgeben

F. hab ich überlegt du hasch nur von de to"chter gsproche

Bei der Sichtung, wer an der Geburtstagsfeier teilgenommen hat und wer nicht, gibt Herr Frahm mit dieser Äußerung das Stichwort, um im Folgenden ausführlich die problematische Abwesenheit des Schwiegersohns zu besprechen. Dass dieser sein Nichtkommen angekündigt hatte, ist $\mathrm{F}$ dabei durchaus bekannt ( $a: h \downarrow$ des * isch also dabei geblie"be d der christian isch ni"ch komme b). Durch das Stichwortgeben wird dem Gesprächspartner in kooperativer Weise Raum gegeben, um eine bestimmte Thematik zu entfalten. Er wird allerdings auch in gewisser Weise dazu verpflichtet.

Auch im folgenden Ausschnitt bemüht sich Herr Frahm kooperativ, Herm Kellner durch ein konkretisierendes Stichwort auszuhelfen (über ein geländespiel am feldherg oder so). Fs Annahme erweist sich allerdings als voreilig, so dass der Versuch misslingt und im Folgenden eine interaktiv aufwändige Richtigstellung erfolgen muss.

\footnotetext{
KF: mein lieber mann (sitzt da so) war mi"tternacht $\downarrow$ *

$\mathrm{KF}$ : hatte ne au"fzeichnung gemacht von der hi"tlerjugend $\uparrow$

KM: da läuft ne se"rie bei * ar * te * glaub ich $\downarrow_{\text {. }}$

F: überde/

F: $\quad$ ein geländespiel am fe"ldberg $>$ oder so $\downarrow<$ nit

KM: (mit)/ snee nee nee" $>$ karll

KM: das/ nee das ni"ch $\downarrow$ ja is da au"ch was noch $\downarrow$
} 
Über die genannten Formen kommunikativer Kooperativität hinaus zeigt sich die verstärkte Partner- und Personenorientienung in dem Gespräch ferner durch die Demonstration einer intensiven Anteilnahme an anderen Personen und in einer deutlichen Praferenz für Gemeinschafilichkeit und Sozialitä gegenüber einer individuellen Profilierung und Abgrenzung. Die Gemeinschaftlichkeit wird dabei durch verschiedene Verfahren im gemeinsamen miteinander Sprechen erzeugt.

Im untersuchten Gesprăch zeigt sich die Anteilnahme von Herm Frahm in seinem intensiven Interesse am Ablauf der Feierlichkeiten des 75. Geburtstag. Durch Nachfragen und die Demonstration von Interesse initiiert er immer wieder detaillierende Darstellungen und expandiert so die Behandlung dieser Thematik.

Die Bevorzugung von Gemeinschaftlichkeit und Sozialität findet u.a. auch Ausdruck in einer Präferenz für die Demonstration von Gemeinsamkeil von Wissen. Man kann vermuten, dass die Demonstration von gemeinsamem Wissen als Demonstration von Gemeinschaftlichkeit für ältere Menschen eine besondere Bedeutung hat. Gemeinsames Wissen hat auch in dem behandelten Tischgespräch einen hohen Stellenwert. Herr Frahm weiß vorab schon viel von dem, was erzählt wird (des programm (hab ich mitgekriegt); hasch du mir was davoo mal gsa"gi (). U.a. dies ermöglicht ihm auch, als Stichwortgeber zu fungieren. Wenn F etwas nicht weiB, führt dies zu Nachfragen, ob er das wissen müsste und zu Bearbeitungen solcher Wissensunterschiede:

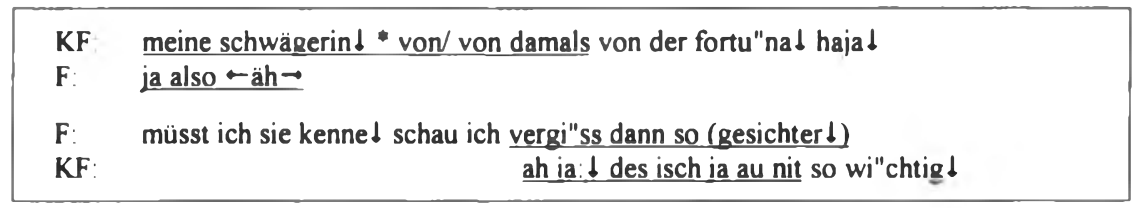

Frau Kellner unterstellt Herm Frahm, dass er ihre Schwägerin kennt. F gibt aber mit Anzeichen von Verlegenheit zu erkennen, dass dies nicht der Fall ist, woraufhin KF die Bedeutsamkeit dieses Wissens herunterstuft. Ganz analog ist der folgende Fall:

\begin{tabular}{|c|c|}
\hline $\mathbf{F}$ & aber die muss ich ni"t kenne $\downarrow \leq \mathrm{LACHT}>$ \\
\hline KM: & nein nein nein nein nein nein nein nein $\downarrow$ \\
\hline $\mathrm{KF}$ & nein die musch du ni"t kennel \\
\hline
\end{tabular}

In diesem und in anderen Gesprächen wird häufig etwas erzählt oder berichtet, was allen Beteiligten bekannt ist. Es geht dabei also nicht um die Mitteilung von Neuem, sondem um die Zelebrierung von etwas gemeinsam Geteiltem. Hier liegt möglicherweise eine Erklärung für das Erwachsenenstereotyp, dass ältere Menschen sich hăufig 
wiederholen. Vor diesem Hintergrund wird auch verständlich, dass kommunikative Verfahren des Wissensausgleichs wie Erzählungen oder Klatsch für ältere Menschen eine besondere Bedeutung haben.

Die hier dargestellten Phänomene sind natürlich nicht auf Gespräche alter Menschen beschränkt. Sie treten - unabhängig vom Alter der Beteiligten - in allen Gesprächen auf, in denen Kooperativität und Gemeinschaftlichkeit eine zentrale Rolle spielen, z.B. in geselligen Runden. Was sie in Gesprächen älterer Menschen auffallig macht, ist die Häufung der Verfahren und die Frequenz, mit der Realisierungen dieser Verfahren auftreten. Ich möchte auch nicht behaupten, dass diese Phänomene für alle alten Menschen charakteristisch sind. Dafür ist das Alter viel zu heterogen, und es gibt große Gruppen, die zu diesen Formen von Kooperativität und Partnerorientierung nicht in der Lage sind, die dazu keine Gelegenheit haben oder denen daran nicht gelegen ist.

\section{Zusammenfassung}

Beginnt man rekapitulierend bei den Daten, so waren ein außergewöhnliches $\mathrm{Maß}$ an Partner- bzw. Personenorientierung und an kommunikativer Kooperativität sowie zahlreiche Episoden der Demonstration von Kompetenz und Normalität hervorstechende Merkmale des untersuchten Gesprächs. Vor diesem Hintergrund ist die Hypothese zu bedenken, dass viele der alterstypisch wirkenden sprachlich-kommunikativen Phänomene sich einer stärkeren Personen- und Beziehungsorientierung der älteren Menschen verdanken, die im Kontrast zu der vorherrschenden Aufgaben- und Sachorientierung der mittleren Generation steht und die aus dieser Perspektive daher besonders auffallig erscheint. In den Analysen wurde zugleich eine starke Orientierung der älteren Generation an den Normalitätsstandards und der Identität der mittleren Generation deutlich, die sich in einer Vielzahl von Kontrastienungen und Demonstrationen des (noch) Teilhabens an dieser ,Normalität' manifestierte.

Diese Phänomene fügen sich zusammen, wenn man sie als Elemente eines Diskurses versteht, in dem Altersidentität entwickelt und fortgeschrieben wird. Für diesen Diskurs ist konstitutiv, dass zum einen Nachweise geführt werden, dass Aspekte der Erwachsenenidentität unbeeinträchtigt fortbestehen, dass zum anderen Abweichungen und Beeinträchtigungen der Erwachsenenidentität thematisiert werden und dass letztlich eigenständige Aspekte von Altersidentität präsentiert werden. Die Ausbildung und Bearbeitung von Altersidentität erfolgt dabei also in einer deutlichen Abhängigkeit von der Erwachsenenidentität. 


\section{Danksagung}

Für eine ausführliche Analyse dieses Gesprächs, in der viele der hier vorgestellten Ideen gemeinsam entwickelt wurden, danke ich Daniela Heidtmann und Reinhold Schmitt Den Alten von Greoux (Provence) danken wir für Inspirationen

\section{Literatur}

Androutsopoulos. J (2001). Von fett zu fabelhaft: Jugendsprache in der Sprachbiografie Osnabrücker Beiträge zur Sprachtheorie, 62, 55-78.

Coupland, N., Coupland, J \& Giles, H (1991). Language, society and the elderly: Discourse, identity and ageing. Oxford: Blackwell.

Fiehler, R (1990) Kommunikation und Emotion. Theoretische und empinsche Untersuchungen zur Rolle von Emotionen in der verbalen Interaktion. Berlin: de Gruyter.

Fiehler. R. (1997). Kommunikation im Alter und ihre sprachwissenschaftliche Analyse. Gibt es einen Kommunikationsstil des Alters? In: M. Selting \& B. Sandig (Eds), Sprech- und Gesprachssile (pp 345-370). Berlin: de Gruyter.

Fiehler, R (1999) Was tut man, wenn man ,kooperativ“ ist? Eine gesprächsanalytische Explikation der Konzepte ,Kooperation' und ,Kooperativität'. In: A. Mönnich \& E.W. Jaskolski (Eds.), Kooperation in der Kommunikation. Festschrifi für Elmar Barlsch (pp. 52-58). München: Reinhardt.

Fiehler, R (2001). Der Stil des Alters. In: I. Keim \& W. Schütte (Eds), Soziale Welten und kommunikative Stile. Festschrift fur Werner Kallmeyer zum 60. Geburtstag. Tübingen: Narr.

Fiehler, R. (2002). Spracherwerb im Enwachsenenalter. In: G. Rickheit, Th. Herrmann \& W Deutsch (Eds), Psycholinguistik. Ein internationales Handbuch Berlin: de Gruyter (im Druck)

Holstein, J A \& Gubrium, J F (2000) The self we live by. Narrative identity in a posimodern world. New York: Oxford University Press

Keupp. H., Ahbe, T., Gmür. W., Höfer, R, Mitzscherlich, B., Kraus, W \& Straus, F. (1999) Identitatskonstruktionen. Das Patchwork der Identitaten in der Spatmoderne. Reinbek: Rowohlt.

Kraus. W (1996). Das erzahlte Selbst. Die narrative Konstruktion von Identität in der Spätmoderne. Pfaffenweiler: Centaurus.

Luckmann, Th. (1988). Kommunikative Gattungen im kommunikativen ,Haushalt' einer Gesellschaft In: G Smolka-Koerdt, P.M. Spangenberg \& D Tillmann-Bartylla (Eds), Der Ursprung der Literatur (pp 279-288). München: Fink

Ryan, E.B \& Kwong See, S.T (1998). Sprache, Kommunikation und Altern In: R. Fiehler \& C. Thimm (Eds), Sprache und Kommunikation im Alter (pp 57-71) Opladen: Westdeutscher Verlag.

Sachweh, S. (2001). „Is doch schön, nech?“ Gesprächsstrategien älterer Menschen. Osnabrücker Beitrage zur Sprachtheorie, 62. 127-149

Schiffrin. D. (1996). Narrative as self-portrait: sociolinguistic constructions of identity. Language in Sociery, 25, 167-203

Schmitt. R (1997) Unterstützen im Gespräch Zur Analyse manifester Kooperationsverfahren Zeitschrift fü Sprachwissenschaft. 16, 52-82.

Schwitalla, J. (1992). Über einige Weisen des gemeinsamen Sprechens. Zeitschrifi für Sprachwissenschaft. 11, 51-73

Schwitalla. J. (1994) Dic Vergegenwärtigung einer Gegenwelt. Sprachliche Formen der sozialen Abgrenzung einer Jugendlichengruppe in Vogelstang. In W. Kallmeyer (Ed.), Kommunikation in der Stadı I (pp 467-509). Berlın de Gruyter 


\section{Transkriptionszeichen}

$1 \quad$ fallende Intonation

$i \quad$ steigende Intonation

" auffällige Bctonung

Dehnung

Verschleifung

$<$ Wort $>$

$>$ Wort<

$\leftarrow$ Wort $\rightarrow$

$\rightarrow$ Wort lauter gesprochen

leiser gesprochen

langsamer gesprochen

schneller gesprochen

\begin{tabular}{ll}
- & kurze Pause \\
(ja) & vermuteter Wortlaut \\
(..) & unverständliche Passage \\
mul/ & Abbruch \\
|aber ja & gleichzeitiges Sprechen \\
\hline nein nie &
\end{tabular}

LACHEN

\#\#
Kommentar der Transkribendin

Erstreckung des Kommentars 